\title{
EDITORIAL
}

\section{Emerging orthopaedics resident training programs, changing paradigms. This is the future.}

Currently, the orthopaedic resident training is based on an apprenticeship model. Residents improve their surgical skills by performing surgeries under the guidance of experienced surgeons. In many cases, residents lack the experience of working under several attending physicians, which may result in the narrowing of their surgical knowledge. Nowadays, many of the orthopaedic residents tend to subspecialize before passing through the whole surgical panel of orthopaedic surgery and traumatology.

In the past, because of working long hours, residents were exposed to and had to perform many procedures under the supervision of different attending surgeons. The attending surgeons had less concern about legal ramifications regarding possible complications and also the hospitals tolerated longer procedure times.

A resident who works his entire learning period under only one attending surgeon represents the current model. For five years, the exposure is only to the cases that his attending chooses or likes to operate. Other methods currently used are represented by meeting presentations, observation during surgery, online videos, all of which being an important part of the training, but not sufficient.

Unfortunately, resident training centers do not offer the same display when it comes to teaching capabilities. For example, there are training centers where standard or key orthopaedic surgeries are not performed anymore. Not at present but in the future, we could find ourselves in the context in which a young surgeon would be theoretically capable of performing major surgeries without knowing to perform basic procedures. This scenario is most probably theoretical than practical, the reality being that at the end of the training, most residents will not be able to perform either of the two. It would be foolish of us to think that a young surgeon could do total hips or knees without learning first to perform simpler surgical procedures.

Changes are occurring in the Romanian National Health Education System, but when it comes 
to training centers, the need of consistency, equality, and equability should be a matter of national concern. There is no debate that an experienced surgeon should not perform more than several procedures, but that is not the case of training residents, who should cover and be exposed to all orthopaedic surgery and traumatology. Through his learning period, an orthopaedic resident should attend a rotational schedule with several attending surgeons so that the exposure is maximal and at the end of which he could perform key procedures. Perhaps, after that, the concept of fellowship programs should be introduced in our country. By attending a subspecialty fellowship, under the supervision of designated experienced surgeons in one field, one can be "certified" that he/ she is well trained. Only after such training, could young surgeons choose to carry out only several types of procedures.

An important part of the educational process of young surgeons is their support in the publishing process. From an objective point of view, there are major differences between the training centers regarding the number of annual published papers and above this, their scientific quality. Publishing a state of the art original paper or a review in a distinguished international journal should be a mandatory step at the end of the training program for all young surgeons. This important step is feasible only in a center that provides the essential setting and all the resources.

In conclusion, the homogeneity of the teaching programs throughout the same university centers and throughout the country is a very important step in reaching high education standards and for increasing patient satisfaction and outcomes.

\section{Conflict of Interest statements}

Authors state no conflict of interest.

Editor in Chief,

Prof. Cătălin Cîrstoiu, MD, PhD

Deputy/Managing editor

Bogdan Crețu, MD, PhD 